Pacific Journal of Mathematics

ENERALIZED CLIFFORD-LITTLEWOOD-ECKMANN
GROUPS II: LINEAR REPRESENTATIONS AND

TARA LYNN SMITH 


\title{
GENERALIZED CLIFFORD-LITTLEWOOD-ECKMANN GROUPS II: LINEAR REPRESENTATIONS AND APPLICATIONS
}

\author{
TARA L. SMITH
}

This paper presents applications of the decomposition of generalized Clifford-Littlewood-Eckmann groups, or CLE-groups, which are given by presentations of the type

$$
\begin{aligned}
\mathbf{G}=\left\langle\omega, a_{1}, \ldots, a_{r}\right| \omega^{n}=1, a_{i}^{n}=\omega^{e(i)} \forall i, \\
\left.a_{i} a_{j}=\omega a_{j} a_{i} \forall i<j, \omega a_{i}=a_{l} \omega \forall i\right\rangle .
\end{aligned}
$$

We begin by studying the irreducible complex representations of the "building block groups" of orders $n^{2}$ and $n^{3}$, and how the representations for the composite groups are constructed from them. This of course also gives a complete set of inequivalent irreducible matrix representations for the generalized Clifford algebras corresponding to these groups. We apply these representation-theoretic results to determine the size of the maximal abelian subgroups of these groups, and to present a generalization of a result of Littlewood on maximal sets of anticommuting matrices. In the final section we consider an alternative generalization of the CLE-groups, in which we require $a_{i}^{n}=1$, but allow $a_{i} a_{j}=\omega^{k} a_{j} a_{i}$ for fixed $k$ dividing $n$, where possibly $k>1$. The irreducible complex representations of these groups are then calculated.

Introduction. These representations have been studied from the standpoint of projective representations of $(\mathbb{Z} / n \mathbb{Z})^{r}$ in [S-I]. However we feel that the presentation given here is somewhat clearer. The results again are of interest to physicists in a number of applications (see [S-I], [Kw]). Throughout this paper the notation and conventions will follow those of [Sm1], [Sm2]. The results of [Sm2] concerning the explicit decomposition of the groups will be used extensively here. The corresponding generalized Clifford algebras are studied in [Sm3].

1. Linear representations. The first application of the decomposition results obtained in [Sm2] is the determination of the irreducible complex representations for the generalized group $\mathbf{G}=\left\langle\omega, a_{1}, \ldots, a_{r}\right|$ $\left.\omega^{n}=1, a_{i}^{n}=\omega^{e(i)} \forall i, a_{i} a_{j}=\omega a_{j} a_{i} \forall i<j, \omega a_{i}=a_{i} \omega \forall i\right\rangle$. To begin we need some general facts about representations of finite groups, which are found in [I]. 
Proposition 1.1. Let $\mathbf{G}$ and $\mathbf{H}$ be finite groups, and let $\operatorname{Irr}(\mathbf{G})$ and $\operatorname{Irr}(\mathbf{H})$ denote their respective sets of irreducible $\mathbb{C}$-representations (and sometimes by abuse of notation, the sets of characters corresponding to the irreducible representations). Then the following hold for all $S \in$ $\operatorname{Irr}(\mathbf{G})$ and $T \in \operatorname{Irr}(\mathbf{H})$ :

(1) $S(g)$ is a scalar multiple of the identity matrix $\forall g \in \mathbf{Z}(\mathbf{G})$.

(2) $\operatorname{Irr}(\mathbf{G} \times \mathbf{H})=\{S \otimes T: S \in \operatorname{Irr}(\mathbf{G}), T \in \operatorname{Irr}(\mathbf{H})\}$.

(3) Let $\mathbf{N}$ be a normal subgroup of $\mathbf{G}$, and suppose $\mathbf{N} \subseteq \operatorname{ker}(S)$. Then $S^{\prime}$ defined by $S^{\prime}(g \mathbf{N})=S(g)$ is an irreducible representation of $\mathbf{G} / \mathbf{N}$. Moreover, any irreducible representation of $\mathbf{G} / \mathbf{N}$ arises in this way.

(4) Let $g \rightarrow \chi(g):=\operatorname{tr} R(g)$ be the character associated with a representation $R$ of $\mathbf{G}$. Then $R$ is irreducible if and only if $[\chi, \chi]:=$ $|\mathbf{G}|^{-1} \sum_{g \in \mathbf{G}} \chi(g) \chi\left(g^{-1}\right)=1$.

(5) Two irreducible representations are distinct (i.e. not similar) if and only if their corresponding characters are distinct.

(6) $|\operatorname{Irr}(\mathbf{G})|$ equals the number of conjugacy classes of $\mathbf{G}$, and $\sum_{\chi \in \operatorname{Irr}(\mathbf{G})} \chi(1)^{2}=|\mathbf{G}|$.

Applying these results to our groups, we get the following proposition, which reveals how to determine the representation theory for an arbitrary group of this type from that of the building block groups (those of order $n^{2}$ or $n^{3}$ ). Recall that if $\mathbf{G}$ and $\mathbf{H}$ are two groups in the category whose objects are finite groups with a distinguished central element $\omega$ of order $n$ and whose morphisms are $\omega$-preserving homomorphisms, we may define

$$
\mathbf{G} \dot{\times} \mathbf{H}=\mathbf{G H}:=\frac{\mathbf{G} \times \mathbf{H}}{\left(\left\langle\omega, \omega^{-1}\right\rangle\right)},
$$

a central product of two such groups, which again has a distinguished central element $\omega=(\omega, 1)=(1, \omega)$ of order $n$.

Proposition 1.2. Let $\mathbf{G}$ and $\mathbf{H}$ be two groups in the category of finite groups with distinguished central elements of order $n$. Let $\omega_{\mathbf{G}}$ and $\omega_{\mathbf{H}}$ denote respectively the distinguished central elements of order $n$ for these two groups. Then $\operatorname{Irr}(\mathbf{G H})=\{S \otimes T: S \in \operatorname{Irr}(\mathbf{G}), T \in$ $\operatorname{Irr}(\mathbf{H})$, and $\left.S\left(\omega_{\mathbf{G}}\right)=T\left(\omega_{\mathbf{H}}\right)\right\}$. 
We have seen [Sm2] that any generalized CLE group decomposes as a product of groups of the type $\mathbf{G}(d, d):=\langle\omega, a, b| \omega^{n}=1$, $a^{n}=b^{n}=\omega^{d}, a b=\omega b a, \omega$ central $\rangle$, where $d$ divides $n$, and at most one group of the type $\mathbf{G}(e):=\left\langle\omega, a \mid \omega^{n}=1, a^{n}=\omega^{e}\right\rangle$, where $e$ divides $n$. (And in fact, for all but at most one of the factors $\mathbf{G}(d, d)$ we may take $d=n$.) Our task now becomes that of determining the irreducible $\mathbb{C}$-representations for the groups $\mathbf{G}(d)$ and $\mathbf{G}(d, d)$, for $d$ dividing $n$. The groups of order $n^{2}$ are quite easy, because they are abelian. We have the result below.

Proposition 1.3. $\mathbf{G}(d)=\langle\omega, a\rangle \cong \mathbb{Z} /\left(n^{2} / d\right) \mathbb{Z} \times \mathbb{Z} / d \mathbb{Z}$ has $n^{2}$ distinct irreducible representations, each of degree one. They are given by sending $\omega$ to any nth root of $1, \xi$, in $\mathbb{C}$ and sending a to any $n^{2} / d$ th root of $1, \zeta$, satisfying $\zeta^{n}=\xi^{d}$.

Finding all the irreducible representations for the groups of order $n^{3}$ is somewhat more complicated. First we must try to determine the number of conjugacy classes in $\mathbf{G}=\mathbf{G}(d, d)$, so that we know how many representations there should be.

Proposition 1.4. The total number of conjugacy classes in the group $\mathbf{G}(d, d)=\langle\omega, a, b\rangle$ is $\sum_{c / n} n^{2} \phi(c) / c^{2}$, where $\phi(c)$ denotes the number of positive integers less than $c$ which are relatively prime to $c$ (i.e. the number of units in $\mathbb{Z} / c \mathbb{Z}$ ). In particular, this number is independent of $d$, which is not a priori clear.

Proof. We begin by determining the number of conjugates of a given element $g \in \mathbf{G}$, and deriving an initial formula for the number of conjugacy classes. We then show that this formula is multiplicative, and derive a simpler form in the case $n$ is a prime power. This allows us finally to give the number of conjugacy classes in the form above. Let $g=\omega^{k} a^{i} b^{j}$. Then the conjugates of $g$ are $\{\alpha g:[h, g]=\alpha$ for some $h \in \mathbf{G}\}$, and the set $\{\alpha:[h, g]=\alpha\}$ is exactly $\left\langle\omega^{c}\right\rangle$, where $c=$ g.c. d. $(i, j, n)$. (This follows from the fact that $\left[a^{i} b^{j}, a^{k} b^{m}\right]=$ $\omega^{i m-j k}$.) Thus $g$ has $n / c$ conjugates, and $g, \omega g, \ldots, \omega^{c-1} g$ will be in different conjugacy classes of $\mathbf{G}$. Notice that $n / c$ is precisely the order of the image of $g$ in $\mathbf{G} / \mathbf{G}^{\prime}$, which is isomorphic to $\mathbb{Z} / n \mathbb{Z} \times$ $\mathbb{Z} / n \mathbb{Z}$. Looking at all elements $g \in \mathbf{G}$, we see that the number of 
conjugacy classes in $\mathbf{G}$ is

$$
f(n):=\sum_{c \mid n}[\text { the number of elements of order } c
$$

in $\mathbb{Z} / n \mathbb{Z} \times \mathbb{Z} / n \mathbb{Z}] n / c$.

Next we wish to show $f(n)$ is multiplicative, i.e. we want to show $f(m) f(n)=f(m n)$, for $m, n$ relatively prime. To see this, first note that the number of elements of order $c$ in $\mathbb{Z} / n \mathbb{Z} \times \mathbb{Z} / n \mathbb{Z}$ is precisely the number of elements of order $c$ in $\mathbb{Z} / c \mathbb{Z} \times \mathbb{Z} / c \mathbb{Z}$. Then

$$
\begin{array}{r}
f(m) f(n)=\left(\sum_{e \mid m}[\# \text { of elts. of order } e \text { in } \mathbb{Z} / e \mathbb{Z} \times \mathbb{Z} / e \mathbb{Z}] m / e\right) \\
\cdot\left(\sum_{c \mid n}[\# \text { of elts. of order } c \text { in } \mathbb{Z} / c \mathbb{Z} \times \mathbb{Z} / c \mathbb{Z}] n / c\right) \\
=\sum_{e \mid m} \sum_{c \mid n}(\# \text { of elts. of order } e \text { in } \mathbb{Z} / e \mathbb{Z} \times \mathbb{Z} / e \mathbb{Z}) \\
\cdot(\# \text { of elts. of order } c \text { in } \mathbb{Z} / c \mathbb{Z} \times \mathbb{Z} / c \mathbb{Z}) \frac{m n}{c e} .
\end{array}
$$

Now

$$
\begin{aligned}
\text { g. c. d. }(m, n) & =1 \Rightarrow \text { g.c. d. }(c, e)=1 \Rightarrow(\mathbb{Z} / e \mathbb{Z})^{2} \times(\mathbb{Z} / c \mathbb{Z})^{2} \\
& \cong(\mathbb{Z} / c e \mathbb{Z})^{2}
\end{aligned}
$$

and an element of order $c e$ in $(\mathbb{Z} / c e \mathbb{Z})^{2}$ must come from an element of order $c$ in $(\mathbb{Z} / c \mathbb{Z})^{2}$ and element of order $e$ in $(\mathbb{Z} / e \mathbb{Z})^{2}$. Therefore

$$
\begin{aligned}
f(m) f(n) & =\sum_{c e \mid m n}\left(\# \text { of elts. of order ce in }(\mathbb{Z} / c e \mathbb{Z})^{2}\right) \frac{m n}{c e} \\
& =f(m n) .
\end{aligned}
$$

Hence it will be sufficient to calculate $f(n)$ for $n=p^{r}, p$ a prime.

The number of elements of order $p^{k}$ in $\mathbb{Z} / p^{k} \mathbb{Z} \times \mathbb{Z} / p^{k} \mathbb{Z}$ is the number of pairs $\left(g_{1}, g_{2}\right), g_{i} \in \mathbb{Z} / p^{k} \mathbb{Z}$, with at least one of the $g_{i}$ of order $p^{k}$. There are $\phi\left(p^{k}\right)\left[2 p^{k}-\phi\left(p^{k}\right)\right]$ such pairs. Recall also 
$\phi\left(p^{k}\right)=p^{k}(1-1 / p)$, for $k \geq 1$. Then we see

$$
\begin{aligned}
f\left(p^{r}\right) & =\sum_{k=0}^{r}\left(\# \text { elts. of order } p^{k} \text { in } \mathbb{Z} / p^{k} \mathbb{Z} \times \mathbb{Z} / p^{k} \mathbb{Z}\right) p^{r-k} \\
& =\sum_{k=0}^{r} \phi\left(p^{k}\right)\left[2 p^{k}-\phi\left(p^{k}\right)\right] p^{r-k}=p^{r}+\sum_{k=1}^{r}\left(p^{2 k}-p^{2 k-2}\right) p^{r-k} \\
& =p^{r}+p^{r} \sum_{k=1}^{r} p^{k}-p^{r-2} \sum_{k=1}^{r} p^{k} \\
& =p^{r}+p^{r}\left(\frac{p^{r+1}-p}{p-1}\right)-p^{r-2}\left(\frac{p^{r+1}-p}{p-1}\right) \\
& =p^{r}+\left(p^{r+1}-p\right) p^{r-2}(p+1)=p^{2 r}+p^{2 r-1}-p^{r-1} \\
& =p^{2 r}+p^{r-1}\left(p^{r}-1\right)=p^{2 r}+\frac{p-1}{p-1}\left(p^{r-1}\right)\left(p^{r}-1\right) \\
& =p^{2 r}+p^{r} \frac{p^{r}-1}{p-1}-p^{r-1} \frac{p^{r}-1}{p-1}=p^{2 r}+\sum_{k=1}^{r} p^{2 r-k}-p^{2 r-k-1} \\
& =\sum_{k=0}^{r} p^{2 r-2 k} \phi\left(p^{k}\right)=\sum_{c \mid n} n^{2} \phi(c) / c^{2} .
\end{aligned}
$$

Then since $f$ is multiplicative, as is the expression $\sum_{c \mid n} n^{2} \phi(c) / c^{2}$, we quickly see that in fact $f(n)=\sum_{c \mid n} n^{2} \phi(c) / c^{2}$, as was claimed in the statement of the proposition.

In our next theorem we will construct precisely this many irreducible $\mathbb{C}$-representations for our group G. Specifically, we will find $n^{2} \phi(c) / c^{2}$ representations of degree $c$. Then

$$
\sum_{\chi \in \operatorname{Irr}(\mathbf{G})} \chi(1)^{2}=\sum_{c \mid n} c^{2} n^{2} \phi(c) / c^{2}=n^{2} \sum_{c \mid n} \phi(c)=n^{3}=|\mathbf{G}| .
$$

Since we know $\omega$ must go to $\boldsymbol{\xi} \mathbf{I}_{c}$, where $\xi$ is a (not necessarily primitive) $n$th root of unity in $\mathbb{C}$ and $\mathbf{I}_{c}$ is the $c \times c$ identity matrix, we analyze the representations realized under each possible choice for the image of $\omega$.

THEOREM 1.5. For every $c$ dividing $n$ there are $c^{2} \phi(n / c)$ irreducible $\mathbb{C}$-representations of $\mathbf{G}=\mathbf{G}(d, d)=\langle\omega, a, b\rangle$ which are of degree $n / c$. They are given as follows:

$\omega \rightarrow \xi \mathbf{I}_{n / c}$, where $\xi$ is a primitive $(n / c)$ th root of 1 in $\mathbb{C}$ (there are $\phi(n / c)$ distinct choices for $\xi)$, and setting $\zeta$ to be a fixed primitive $n^{2} / d$ th root of 1 satisfying $\zeta^{n c}=\xi^{d}$, 


$$
\begin{aligned}
& a \rightarrow A^{\prime} \in\left\{A, \zeta^{n / d} A, \ldots, \zeta^{(n / d)(c-1)} A\right\}, \\
& b \rightarrow B^{\prime} \in\left\{B, \zeta^{n / d} B, \ldots, \zeta^{(n / d)(c-1)} B\right\}, \text { where }
\end{aligned}
$$

$$
A=\zeta^{c}\left(\begin{array}{ccccc}
0 & 1 & 0 & \cdots & 0 \\
& 0 & 1 & & \\
& & & \ddots & \\
& & & & 1 \\
1 & 0 & & & 0
\end{array}\right), \quad B=\zeta^{c}\left(\begin{array}{ccc}
1 & & \\
& \xi & \\
& & \ddots \\
& & \xi^{n / c-1}
\end{array}\right)
$$

are $n / c \times n / c$ matrices (so there are $c^{2}$ choices for the images of $a$ and $b)$.

Proof. We have three things to show. We must show that the maps above yield representations for $\mathbf{G}$, that they are irreducible, and that there is no duplication among the different maps (i.e. that they are all distinct representations). If we can prove all of these we will be done, for then the remarks above show that these must be all of the irreducible representations of $\mathbf{G}$. Then let $\mathbf{T}$ be defined by $\mathbf{T}(\omega)=$ $\xi \mathbf{I}_{n / c}, \mathbf{T}(a)=A^{\prime}, \mathbf{T}(b)=B^{\prime}$. It is easy to see that $\mathbf{T}\left(a^{n}\right)=\mathbf{T}\left(b^{n}\right)=$ $\mathbf{T}\left(\omega^{d}\right)=\xi^{d} \mathbf{I}_{n / c}$, and that $\mathbf{T}(a) \mathbf{T}(b)=\mathbf{T}(\omega) \mathbf{T}(b) \mathbf{T}(a)$. Therefore $\mathbf{T}$ is a linear representation of $\mathbf{G}$, i.e. it defines a group homomorphism from $\mathbf{G}$ into $\mathrm{GL}(n / c, \mathbb{C})$.

To show irreducibility, we will look at the character $\chi$ associated to T. Without loss of generality, we may assume for this part of the proof that $A^{\prime}=A$ and $B^{\prime}=B$. Recall that $\chi(g)$ is defined to be the trace of the representation $\mathbf{T}(g)$. Again it is an easy exercise to show that $\chi\left(\omega^{i} a^{j} b^{k}\right)=\operatorname{tr}\left(\xi^{i} A^{j} B^{k}\right)=0$ unless $j \equiv k \equiv 0(\bmod n / c)$. If $j=$ $\alpha n / c, k=\beta n / c, 0 \leq \alpha, \beta \leq c$, then $\chi\left(\omega^{i} a^{j} b^{k}\right)=\left(\xi^{i} \zeta^{n(\alpha+\beta)}\right) n / c$. (Notice that $\xi^{i} \zeta^{n(\alpha+\beta)}$ is an $n$th root of 1.) In particular, $\chi(g) \neq 0$ if and only if $\mathbf{T}(g)$ is a scalar matrix, if and only if the order of the image of $g$ in $\mathbf{G} / \mathbf{G}^{\prime}$ divides $c$. In this case, $\chi(g)=\mu(n / c)$ for some $n$th root of $1, \mu$, and $\chi\left(g^{-1}\right)=\mu^{-1}(n / c)$. Hence $\chi(g) \chi\left(g^{-1}\right)=n^{2} / c^{2}$. Now $\mathbf{T}$ will be irreducible if and only if $[\chi, \chi]=1$. Then

$$
\begin{aligned}
\sum_{g \in \mathbf{G}} \chi(g) \chi\left(g^{-1}\right) & \left.=n\left(n^{2} / c^{2}\right) \text { (\#of elements of order dividing } c \text { in } \mathbf{G} / \mathbf{G}^{\prime}\right) \\
& =n\left(n^{2} / c^{2}\right)=n^{3}=|\mathbf{G}|,
\end{aligned}
$$

and we see that indeed $\mathbf{T}$ is irreducible.

Finally we must show that for each choice of $\xi, A^{\prime}$, and $B^{\prime}$, we actually get distinct representations. It is clear that any two distinct choices for the image of $\omega$ give rise to distinct representations. We 
must then show that different choices for $A^{\prime}$ or $B^{\prime}$ also yield distinct representations, and we will be done. Let $\mathbf{T}_{1}$ and $\mathbf{T}_{2}$ be two of the representations above of size $n / c$, with corresponding characters $\chi_{1}$ and $\chi_{2}$, and assume $\chi_{1}$ and $\chi_{2}$ are equal on $\mathbf{G}$. We will show $\mathbf{T}_{1}=\mathbf{T}_{2}$. First, we may clearly assume that $\mathbf{T}_{1}(\omega)=\mathbf{T}_{2}(\omega)=\xi \mathbf{I}_{n / c}$. Let $\mathbf{T}_{1}(a)=\zeta^{\text {in } / d} A$, and $\mathbf{T}_{2}(a)=\zeta^{j n / d} A, 0 \leq i, j \leq c-1$. We need to show $i=j$. Here $\chi_{1}\left(a^{n / c}\right)=(n / c) \zeta^{n+i(n / c)(n / d)}, \chi_{2}\left(a^{n / c}\right)=$ $(n / c) \zeta^{n+j(n / c)(n / d)}$. Then $i n^{2} / c d \equiv j n^{2} / c d\left(\bmod n^{2} / d\right)$, so $i \equiv j$ $(\bmod c)$ as desired, showing $\mathbf{T}_{1}(a)=\mathbf{T}_{2}(a)$. The same argument shows that if $\chi_{i}\left(b^{n / c}\right)=\chi_{2}\left(b^{n / c}\right)$, then $\mathbf{T}_{1}(b)=\mathbf{T}_{2}(b)$. Thus we get $c^{2} \phi(n / c)$ irreducible representations of size $n / c$, as claimed.

We can now use this information to answer some questions about a general group $\mathbf{G}$ of this type, of order $n^{r+1}$, say. We notice that for $\mathbf{G}(d, d)$ there are $c^{2}$ different irreducible representations sending $\omega$ to a given $n / c$ th root of unity in $\mathbb{C}$, each of degree $n / c$. For $\mathbf{G}(d)$ there are $n$ different choices of irreducible representations for each choice of image of $\omega$, each of degree 1. This gives us the next two corollaries.

Corollary 1.6. Let $\mathbf{G}=\left\langle\omega, a_{1}, \ldots, a_{r}\right| \omega^{n}=1, a_{i}^{n} \omega^{e(i)} \forall i$, $\left.a_{i} a_{j}=\omega a_{j} a_{i} \forall i<j, \omega a_{i}=a_{i} \omega \forall i\right\rangle$. If $r$ is even, $\mathbf{G}$ has $c^{r} \phi(n / c)$ distinct irreducible representations of degree $(n / c)^{r / 2}$ for every $c$ dividing $n$, while if $r$ is odd, $\mathbf{G}$ has $n c^{r-1} \phi(n / c)$ distinct irreducible representations of degree $(n / c)^{(r-1) / 2}$ for every $c$ dividing $n$. In either case, these are all the irreducible representations for $\mathbf{G}$.

COROLlaRY 1.7. Let $\mathbf{G}$ be as in the previous corollary. If $r$ is even, $\mathbf{G}$ has exactly $\sum_{c \mid n} c^{r} \phi(n / c)$ conjugacy classes, while if $r$ is odd, $\mathbf{G}$ has $\sum_{c \mid n} n c^{r-1} \phi(n / c)$ conjugacy classes.

We can also make an observation on the existence of faithful irreducible representations for our groups. We have the following:

Proposition 1.8. Let $\mathbf{G}$ be as above. If $r$ is even, then any irreducible representation of $\mathbf{G}$ of maximal degree $n^{r / 2}$ is faithful, but no others. If $r$ is odd, then $\mathbf{G}$ has a faithful irreducible representation if and only if $Z(\mathbf{G}) \cong \mathbb{Z} / n^{2} \mathbb{Z}$, in which case any irreducible representation of $\mathbf{G}$ of maximal degree $n^{(r-1) / 2}$ is faithful, but no others.

Proof. First observe that the proposition is true for $r=1$ or 2 . Now assume $r$ is even, and that the theorem holds for $r-2$. Let $\mathbf{G}=\mathbf{H K}$, 
where $|\mathbf{H}|=n^{r-1}$ and $|\mathbf{K}|=n^{3}$. We must show that for $T \in \operatorname{Irr}(\mathbf{H})$ and $S \in \operatorname{Irr}(\mathbf{K}), T \otimes S \in \operatorname{Irr}(\mathbf{H K})$ is faithful if and only if $T$ and $S$ are. In other words, we must show that the kernel of $T \otimes S$ on $\mathbf{H} \times \mathbf{K}$ is exactly $\left\langle\left(\omega_{\mathbf{H}}, \omega_{\mathbf{K}}^{-1}\right)\right\rangle$. Recall that the character $\chi$ associated to $T \otimes S$ is given by $\chi((h, k))=\chi_{T}(h) \chi_{S}(k)$, where $\chi_{T}$ and $\chi_{S}$ are the characters associated to $T$ and $S$ respectively. Then $T \otimes S$ is faithful on $\mathbf{H K}$ if and only if the only elements $(h, k)$ for which $\chi((h, k))=\chi_{T}(1) \chi_{S}(1)$ are in $\left\langle\left(\omega_{\mathbf{H}}, \omega_{\mathbf{K}}^{-1}\right)\right\rangle$. Assume $T$ and $S$ are faithful. Then $\chi((h, k))=\chi_{T}(1) \chi_{S}(1)$ if and only if $T(h)=\mu \mathbf{I}_{n^{(r-2) / 2}}$ and $S(k)=\mu^{-1} \mathbf{I}_{n}$ for some $n$th root of $1, \mu$. Faithfulness of $T$ and $S$ then implies $h=\left(\omega_{\mathbf{H}}\right)^{p}$ and $k=\left(\omega_{\mathbf{K}}\right)^{q}$ for some $p$ and $q$. Then since $T \otimes S$ is a representation on $\mathbf{H K}$, and $T\left(\omega_{\mathbf{H}}\right)^{p}=\mu \mathbf{I}_{n^{(r-2) / 2}}$, $S\left(\omega_{\mathbf{K}}\right)^{q}=\mu^{-1} \mathbf{I}_{n}$, we must in fact have $q=-p$. This is what we wished to show. Next assume $r$ is odd. Then we can write $\mathbf{G}=\mathbf{H K}$ where now $|\mathbf{H}|=n^{r}$ and $|\mathbf{K}|=n^{2}$. The same argument as given above holds, except that now $S(k)=\mu^{-1} \in \mathbb{C}$. Conversely, if either $T$ or $S$ is not faithful, then of course $T \otimes S$ cannot be faithful.

2. Maximal Abelian subgroups and Littlewood's problem. In this section we discuss two applications of the representation theory for these groups which we examined in the last section. In particular we use knowledge of the irreducible representations to determine the size of maximal abelian subgroups of our groups, and we also use the representations of the groups to determine the maximal sizes of collections of matrices satisfying certain properties.

LEMMA 2.1. Let $\mathbf{H}$ be any subgroup of $\mathbf{G}=\left\langle\omega, a_{1}, \ldots, a_{r}\right| \omega^{n}=1$, $\left.a_{i}^{n}=\omega^{e(i)} \forall i, a_{i} a_{j}=\omega a_{j} a_{i} \forall i<j, \omega a_{i}=a_{i} \omega \quad \forall i\right\rangle$ containing $\mathbf{Z}(\mathbf{G})$, and let $r=2 i$ or $2 i+1$. If $[\mathbf{G}: \mathbf{H}]=n^{2 i} / m$ for some $m$, then $\left[\mathbf{G}: C_{\mathbf{G}}(\mathbf{H})\right] \leq m$.

Proof. For any $g \in \mathbf{G}$, let $g^{\prime}$ denote its image in $\mathbf{G} / \mathbf{Z}(\mathbf{G})$. Observe that if $\left\{h_{1}^{\prime}, h_{2}^{\prime}, \ldots, h_{k}^{\prime}\right\}$ are generators for $\mathbf{H} / \mathbf{Z}(\mathbf{G})$, then $\{\mathbf{Z}(\mathbf{G})$, $\left.h_{1}, h_{2}, \ldots, h_{k}\right\}$ will generate $\mathbf{H}$. Furthermore, since $\mathbf{H} / \mathbf{Z}(\mathbf{G})$ is abelian, there exists a set of generators $\left\{h_{1}^{\prime}, h_{2}^{\prime}, \ldots, h_{k}^{\prime}\right\}$ for it such that the order of $\mathbf{H} / \mathbf{Z}(\mathbf{G})$ is exactly the product of the orders of the generators $h_{i}^{\prime}$. This order is, by hypothesis, just $m$. Since $\left\{\mathbf{Z}(\mathbf{G}), h_{1}, h_{2}, \ldots, h_{k}\right\}$ generates $\mathbf{H}$, we see that $C_{\mathbf{G}}(\mathbf{H})=$ $\bigcap C_{\mathbf{G}}\left(h_{i}\right)$, and so $\left[\mathbf{G}: C_{\mathbf{G}}(\mathbf{H})\right] \leq \prod_{i=1}^{k}\left[\mathbf{G}: C_{\mathbf{G}}\left(h_{i}\right)\right]$. On the other hand, [G: $\left.C_{\mathbf{G}}\left(h_{i}\right)\right]$ is just the number of conjugates of $h_{i}$ in $\mathbf{G}$. An easy generalization of the argument in (1.4) shows that this in turn is 
just the order of $h_{i}^{\prime}$ in $\mathbf{H} / \mathbf{Z}(\mathbf{G})$. In particular, [G: $\left.C_{\mathbf{G}}(\mathbf{H})\right] \leq m$, as claimed.

TheOREM 2.2. Let $\mathbf{A}$ be a maximal abelian subgroup of $\mathbf{G}$, where $|\mathbf{G}|=n^{r+1}$, and $r=2 i$ or $2 i+1$. Then $[\mathbf{G}: \mathbf{A}]=n^{i} ;$ in other words [G: A] is exactly the maximal dimension of an irreducible complex representation of $\mathbf{G}$.

Proof. Let $[\mathbf{G}: \mathbf{A}]=n^{2 i} / m$. Since $\mathbf{A}$ is maximal, clearly $\mathbf{A} \supseteq$ $\mathbf{Z}(\mathbf{G}) \supseteq \mathbf{G}^{\prime}$, and so $\mathbf{A}$ is normal. Then by Itô's Theorem in group representation theory [CR: p. 365], we have $n^{i}[\mathbf{G}: \mathbf{A}]$, so $m \leq n^{i}$. On the other hand, the lemma above shows $\left[\mathbf{G}: C_{\mathbf{G}}(\mathbf{A})\right] \leq m$. Since $\mathbf{A}$ is maximal abelian, we must have $\mathbf{A}=C_{\mathbf{G}}(\mathbf{A})$, and this means $n^{2 i} / m \leq m$. This in turn gives $n^{2 i} \leq m^{2}$, or $n^{i} \leq m$. Combining all this, we see that in fact $m=n^{i}$.

Next we turn our attention to a problem discussed by Littlewood in a paper on sets of anticommuting matrices [Li]. We want to determine the largest size of a set $\left\{B_{1}, \ldots, B_{s}\right\}$ of complex matrices of (fixed) dimension $n^{q} m, m$ prime to $n$, satisfying $B_{i} B_{j}=\omega B_{j} B_{i}, i<j$, and $B_{i}^{n} \in\left\{1, \omega, \ldots, \omega^{n-1}\right\}$, where $\omega$ is a primitive $n$th root of unity in $\mathbb{C}$. Littlewood showed that the answer is $2 q+1$ when $n=2$ or 3; he obtained this result by considering group characters on groups of our type for $n=2$ or 3 . We give here a proof generalizing this result to an arbitrary positive integer $n$, and showing that the maximal size remains $2 q+1$ in all cases.

THEOREM 2.3. The largest integer $s$ such that there exist matrices $B_{1}, B_{2}, \ldots, B_{s}$ of dimension $n^{q} m$ over $\mathbb{C}$, with $m$ prime to $n$, which satisfy the conditions given above, is $s=2 q+1$.

Proof. Let $\mathbf{G}=\left\langle\omega, a_{1}, \ldots, a_{r}\right| \omega^{n}=1, a_{i}^{n}=\omega^{e(i)} \forall i, a_{i} a_{j}=$ $\left.\omega a_{j} a_{i} \forall i<j, \omega a_{i}=a_{i} \omega \forall i\right\rangle$. Let $r=2 q$ or $2 q+1$. An elementary linear algebra argument shows that a maximal set of " $\omega$-commuting" elements in $\mathbf{G}$ is given by $\left\{a_{1}, \ldots, a_{r}\right\}$ if $r=2 q+1$ is odd, and by $\left\{a_{1}, \ldots, a_{r}, a_{1} a_{2}^{-1} a_{3} \cdots a_{r}^{-1}\right\}$ if $r=2 q$ is even. In either case, the maximal set is of order $2 q+1$. An irreducible $\mathbb{C}$-representation for $\mathbf{G}$ sending $\omega$ in $\mathbf{G}$ to $\omega$ in $\mathbb{C}$ will be of dimension $n^{q}$, and a direct sum of $m$ copies of it will then be of the desired dimension $n^{q} m$. The matrices corresponding to the images of the maximal set of $\omega$-commuting elements in $\mathbf{G}$, as given above, will be a set of $2 q+1$ matrices of dimension $n^{q} m$ satisfying the desired conditions. 
Therefore a maximal set is at least this large. Conversely, any set of matrices $B_{1}, \ldots, B_{s}$ satisfying the given conditions will themselves determine a representation for a group $\mathbf{G}=\left\langle\omega, a_{1}, \ldots, a_{s}\right| \omega^{n}=1$, $\left.a_{i}^{n}=\omega^{e(i)} \forall i, a_{i} a_{j}=\omega a_{j} a_{i} \forall i<j, \omega a_{i}=a_{i} \omega \forall i\right\rangle$, under which $\omega$ in $\mathbf{G}$ is sent to $\omega$ in $\mathbb{C}$. This representation must therefore be a direct sum of irreducibles of maximal dimension, which will be $n^{p}$, where $s=2 p$ or $2 p+1$. Since the $B_{i}$ 's are of dimension $n^{q} m$, we must have $p \leq q$. In particular, the largest set of matrices of dimension $n^{q} m$ and satisfying the given conditions contains at most $2 q+1$ elements. This completes our proof.

3. An alternative generalization. We now consider the group $\mathbf{G}$ given by

$$
\begin{aligned}
\mathbf{G}=\left\langle\omega, a_{1}, \ldots, a_{r}\right| \omega^{e}=a_{i}^{n}=1 \forall i, a_{i} a_{j}=\omega a_{j} a_{i} & \forall i<j, \\
\omega a_{i} & \left.=a_{i} \omega \forall i\right\rangle,
\end{aligned}
$$

where $e$ is some (proper) divisor of $n$. The study of the decomposition of such a group follows a similar (but simpler) argument to the one given in [Sm2] for the groups we have been considering up to now. Thus we will leave out most of the details.

Notation. Let $\mathbf{G}_{r}$ be the group as given above, and let $\mathbf{G}_{-r}$ be the group for which $a_{i}^{n}=\omega^{n(n-1) / 2}, 1 \leq i \leq r$. Notice that if $n$ is odd, or if $n$ is even and $2 e$ divides $n$, then $\omega^{n(n-1) / 2}=1$, and $\mathbf{G}_{r} \cong \mathbf{G}_{-r}$. The notation stems from the fact that if $n$ is even and $2 e$ does not divide $n$, then $\omega^{n(n-1) / 2}$ is a central element of order 2, i.e. it is " -1 ".

Proposition 3.1. For $\mathbf{G} \cong \mathbf{G}_{r}$ or $\mathbf{G} \cong \mathbf{G}_{-r}$, the following hold:

(i) $|\mathbf{G}|=n^{r} e$.

(ii) Let $z:=a_{1} a_{2}^{-1} a_{3} \cdots a_{r}^{(-1)^{r+1}}$. If $r$ is odd, then $z$ is central. If $r$ is even, then $z a_{i}=\omega a_{i} z$ for all $i$.

(iii) $z^{n}=\omega^{n(n-1) / 2}$ if $r \equiv 2$ or $3(\bmod 4), z^{n}=1$ if $r \equiv 0$ or $1(\bmod 4)$.

LEMMA 3.2. For $r \geq 2, \mathbf{G}_{r} \cong \mathbf{G}_{-(r-2)} \dot{\times} \mathbf{G}_{2}$ and $\mathbf{G}_{-r} \cong \mathbf{G}_{r-2} \dot{\times} G_{-2}$.

Proof. As in the case for the generalized CLE-groups, observe that if $\mathbf{G}=\left\langle\omega, a_{1}, \ldots, a_{r}\right\rangle$, then $\mathbf{G} \cong\left\langle\omega, x a_{1}, \ldots, x a_{r-2}\right\rangle \dot{\times}\left\langle\omega, a_{r-1}, a_{r}\right\rangle$, where $x=a_{r} a_{r-1}^{-1}$. Then look at the $n$th powers of the given generators. 
LEMMA 3.3.

(i) $\mathbf{G}_{-2} \dot{\times} \mathbf{G}_{-1} \cong \mathbf{G}_{2} \dot{\times} \mathbf{G}_{-1}$.

(ii) $\mathbf{G}_{-2} \dot{\times} \mathbf{G}_{-2} \cong \mathbf{G}_{2} \dot{\times} \mathbf{G}_{2}$.

Proof. In case (i), let $\omega, a, b$ be the generators of $\mathbf{G}_{-2}$, and let $\omega, c$ be the generators of $\mathbf{G}_{-1}$. Let $\mathbf{G}$ denote the central product. Then $\mathbf{G}$ is also generated by $\omega, a c, b c, c$, where $\omega$ and $c$ are central, $a c$ and $b c$-commute, and $(a c)^{n}=(b c)^{n}=1$.

In case (ii), let the first factor $\mathbf{G}_{-2}$ be generated by $\omega, a_{1}, a_{2}$, and the second factor $\mathbf{G}_{-2}$ by $\omega, b_{1}, b_{2}$. Then the product is generated by the four $\omega$-commuting elements $a_{1}, a_{2}, a_{1} a_{2}^{-1} b_{1}, a_{1} a_{2}^{-1} b_{2}$, with $n$th powers being $-1,-1,1,1$ respectively. On the other hand, letting the first factor $\mathbf{G}_{2}$ be generated by $\omega, c_{1}, c_{2}$ and the second factor $\mathbf{G}_{2}$ by $\omega, d_{1}, d_{2}$, we see that the product of these two groups is generated by the four $\omega$-commuting elements $c_{1}, c_{2}$, $c_{1} c_{2}^{-1} d_{1}, c_{1} c_{2}^{-1} d_{2}$, with $n$th powers being $1,1,-1,-1$ respectively. Now if $x, y, z, w$ are $\omega$-commuting elements, then $w, z, y, x$ are $\omega^{-1}$-commuting elements, and the two given groups are isomorphic.

Observation 3.4. It is easily seen that the two groups $\mathbf{G}_{1}$ and $\mathbf{G}_{-1}$ are abelian, with $\mathbf{G}_{1} \cong \mathbb{Z} / n \mathbb{Z} \times \mathbb{Z} / e \mathbb{Z}$, and, in the case when $n$ is even and $2 e$ does not divide $n, \mathbf{G}_{-1} \cong \mathbb{Z} / 2 n \mathbb{Z} \times \mathbb{Z} /(e / 2) \mathbb{Z}$.

THEOREM 3.5 (Decomposition Theorem). If $n$ is odd or if $2 e$ divides $n$, then $\mathbf{G}_{r}$ is isomorphic to $\left(\mathbf{G}_{2}\right)^{r / 2}$ if $r$ is even, and to $\left(\mathbf{G}_{2}\right)^{(r-1) / 2} \mathbf{G}_{1}$ if $r$ is odd. If $n$ is even and $2 e$ does not divide $n$, then the isomorphism type of $\mathbf{G}_{r}$ depends on the class of $r(\bmod 8)$ and is as given below. (Notice the exact parallel with the groups $\mathbf{G}_{0, t}$ in [LS].)

\begin{tabular}{|c|c|c|c|}
\hline$r(\bmod 8)$ & Isomorphism type of $\mathbf{G}_{r}$ & $r(\bmod 8)$ & Isomorphism type of $\mathbf{G}_{r}$ \\
\hline 0 & $\left(\mathbf{G}_{2}\right)^{r / 2}$ & 4 & $\left(\mathbf{G}_{2}\right)^{(r-2) / 2} \mathbf{G}_{-2}$ \\
1 & $\left(\mathbf{G}_{2}\right)^{(r-1) / 2} \mathbf{G}_{1}$ & 5 & $\left(\mathbf{G}_{2}\right)^{(r-3) / 2} \mathbf{G}_{-2} \mathbf{G}_{1}$ \\
2 & $\left(\mathbf{G}_{2}\right)^{r / 2}$ & 6 & $\left(\mathbf{G}_{2}\right)^{(r-2) / 2} \mathbf{G}_{-2}$ \\
3 & $\left(\mathbf{G}_{2}\right)^{(r-1) / 2} \mathbf{G}_{-1}$ & 7 & $\left(\mathbf{G}_{2}\right)^{(r-1) / 2} \mathbf{G}_{-1}$ \\
\hline
\end{tabular}

Now we are ready to consider the linear representations for these groups (which are related to projective representations for $\mathbb{Z} / n \mathbb{Z} \times$ $\mathbb{Z} / n \mathbb{Z}$ ). As before it will suffice to determine the irreducible complex 
representations for the "building block" groups $\mathbf{G}_{1}, \mathbf{G}_{-1}, \mathbf{G}_{2}, \mathbf{G}_{-2}$. The first two, being abelian, require no further comment. For the latter two, we begin by determining the number of conjugacy classes.

Proposition 3.6. The groups $\mathbf{G}_{2}$ and $\mathbf{G}_{-2}$ each have $\sum_{c \mid e} n^{2} \phi(c) / c^{2}$ conjugacy classes.

Proof. Notice the subtle difference between this result and that in (1.4). The proof follows a similar line. Observe that the element $g=\omega^{k} a^{i} b^{j}$ has $e / c$ distinct conjugates, where $c:=\operatorname{gcd}(i, j, e)$. The elements $g, \omega g, \ldots, \omega^{c-1} g$ will all be in different conjugacy classes. Now the number of ordered pairs $(i, j)$ with $0 \leq i, j \leq n-1$ and $\operatorname{gcd}(i, j, e)=c$ is precisely $\left\{\left(n^{2} / e^{2}\right) \cdot\right.$ [the number of elements of order $e / c$ in $\mathbb{Z} / e \mathbb{Z} \times \mathbb{Z} / e \mathbb{Z}]\}$. Thus the total number of conjugacy classes is

$\left(n^{2} / e^{2}\right) \sum_{c \mid e}[$ the number of elements of order $c$ in $\mathbb{Z} / e \mathbb{Z} \times \mathbb{Z} / e \mathbb{Z}] e / c$.

As in the previous proof, we see this is $\left(n^{2} / e^{2}\right) \sum_{c \mid e} e^{2} \phi(c) / c^{2}=$ $\sum_{c \mid e} n^{2} \phi(c) / c^{2}$.

The next theorem will produce precisely this many irreducible $\mathbb{C}$ representations for the groups $\mathbf{G}_{2}$ and $\mathbf{G}_{-2}$. Specifically, we will find $n^{2} \phi(c) / c^{2}$ representations of degree $c$ for each $c$ dividing $e$. Then, summing over all the corresponding irreducible characters $\chi$, we have

$$
\sum_{\chi \in \operatorname{Irr}(\mathbf{G})} \chi(1)^{2}=\sum_{c \mid e} c^{2} n^{2} \phi(c) / c^{2}=n^{2} \sum_{c \mid e} \phi(c)=n^{2} e=|\mathbf{G}| .
$$

Since we know $\omega$ must go to $\boldsymbol{\xi} \mathbf{I}_{c}$, where $\boldsymbol{\xi}$ is a (not necessarily primitive) $e$ th root of unity in $\mathbb{C}$ and $\mathbf{I}_{c}$ is the $c \times c$ identity matrix, we analyze the representations realized under each possible choice for the image of $\omega$.

THEOREM 3.7. For every $c$ dividing $e$ there are $\left(n^{2} c^{2} / e^{2}\right) \phi(e / c)$ irreducible $\mathbb{C}$-representations of degree $e / c$ for $\mathbf{G}=\mathbf{G}_{2}$. They are given as follows:

$\omega \rightarrow \boldsymbol{\xi} \mathbf{I}_{e / c}$, where $\boldsymbol{\xi}$ is a primitive $(e / c)$ th root of 1 in $\mathbb{C}$ (there are $\phi(e / c)$ distinct choices for $\xi)$, and setting $\zeta$ to be a primitive nth root of 1 ,

$a \rightarrow A^{\prime} \in\left\{A, \zeta A, \ldots, \zeta^{n c / e-1} A\right\}$, 


$$
b \rightarrow B^{\prime} \in\left\{B, \zeta B, \ldots, \zeta^{n c / e-1} B\right\} \text {, where }
$$

$$
A=\left(\begin{array}{ccccc}
0 & 1 & 0 & \cdots & 0 \\
& 0 & 1 & & \\
& & & \ddots & \\
& & & & 1 \\
1 & 0 & & & 0
\end{array}\right), \quad B=\left(\begin{array}{llll}
1 & & & \\
& \xi & & \\
& & \ddots & \\
& & \xi^{(e / c-1)}
\end{array}\right)
$$

are $e / c \times e / c$ matrices (so there are $(n c / e)^{2}$ choices for the images of $a$ and $b$ ).

Proof. As in the proof of (1.5), we must show that the maps above yield representations for $\mathbf{G}$, that they are irreducible, and that there is no duplication among the different maps (i.e. that they are all distinct representations). Then let $\mathbf{T}$ be defined by $\mathbf{T}(\omega)=\xi \mathbf{I}_{e / c}, \mathbf{T}(a)=A^{\prime}$, $\mathbf{T}(b)=B^{\prime}$. It is easy to see that $\mathbf{T}\left(a^{n}\right)=\mathbf{T}\left(b^{n}\right)=\mathbf{I}_{e / c}$, and that $\mathbf{T}(a) \mathbf{T}(b)=\mathbf{T}(\omega) \mathbf{T}(b) \mathbf{T}(a)$. Therefore $\mathbf{T}$ is a linear representation of G. To show irreducibility, we will look at the character $\chi$ associated to T. As before, we assume for this part of the proof that $A^{\prime}=A$ and $B^{\prime}=B$. Again it is easy to show that $\chi\left(\omega^{i} a^{j} b^{k}\right)=\operatorname{tr}\left(\xi^{i} A^{j} B^{k}\right)=$ 0 unless $j \equiv k \equiv 0(\bmod e / c)$. If $j=\alpha e / c, k=\beta e / c$, then $\chi\left(\omega^{i} a^{j} b^{k}\right)=\xi^{i} e / c$. In particular, $\chi(g) \neq 0$ if and only if $\mathbf{T}(g)$ is a scalar matrix, if and only if the order of the image of $g$ in $\mathbf{G} / \mathbf{G}^{\prime}$ divides $n c / e$. In this case, $\chi(g)=\xi^{\gamma}(e / c)$ for some $\gamma$, and $\chi\left(g^{-1}\right)=$ $\xi^{-\gamma}(e / c)$. Hence $\chi(g) \chi\left(g^{-1}\right)=e^{2} / c^{2}$. Now $\mathbf{T}$ will be irreducible if and only if $[\chi, \chi]=1$. Then

$$
\begin{aligned}
\sum_{g \in \mathbf{G}} & \chi(g) \chi\left(g^{-1}\right) \\
& =e\left(e^{2} / c^{2}\right) \cdot\left(\# \text { of elements of order dividing } n c / e \text { in } \mathbf{G} / \mathbf{G}^{\prime}\right) \\
& =e\left(e^{2} / c^{2}\right)(n c / e)^{2}=n^{2} e=|\mathbf{G}|,
\end{aligned}
$$

and we see that indeed $\mathbf{T}$ is irreducible. Finally we must show that for each choice of $\xi, A^{\prime}$, and $B^{\prime}$, we actually get distinct representations. We will do this by showing that the characters for each choice take on different values for some element of $\mathbf{G}$. First, it is slear that any two distinct choices for the image of $\omega$ give rise to distinct representations. We must then show that different choices for $A^{\prime}$ or $B^{\prime}$ also yield distinct representations, and we will be done. Let $T_{1}$ and $T_{2}$ be two of the representations above of size $e / c$, with corresponding characters $\chi_{1}$ and $\chi_{2}$, and assume $\chi_{1}$ and $\chi_{2}$ are equal on $\mathbf{G}$. We will show $\mathbf{T}_{1}=\mathbf{T}_{2}$. First, we may clearly assume 
that $\mathbf{T}_{1}(\omega)=\mathbf{T}_{2}(\omega)=\xi \mathbf{I}_{e / c}$. Let $\mathbf{T}_{1}(a)=\zeta^{j} A$, and $\mathbf{T}_{2}(a)=\zeta^{k} A$. We need to show $j \equiv k(\bmod n c / e)$. Here $\chi_{1}\left(a^{e / c}\right)=(e / c) \zeta^{j e / c}$, $\chi_{2}\left(a^{e / c}\right)=(e / c) \zeta^{k e / c}$. Therefore $j e / c \equiv k e / c(\bmod n)$. Then $j \equiv k$ $(\bmod n c / e)$ as desired, showing $\mathbf{T}_{1}(a)=\mathbf{T}_{2}(a)$. The same argument shows that if $\chi_{1}\left(b^{e / c}\right)=\chi_{2}\left(b^{e / c}\right)$, then $\mathbf{T}_{1}(b)=\mathbf{T}_{2}(b)$. Thus we get $(n c / e)^{2} \phi(e / c)$ irreducible representations of size $e / c$, as claimed.

THEOREM 3.8. For every $c$ dividing $e$ there are $\left(n^{2} c^{2} / e^{2}\right) \phi(e / c)$ irreducible $\mathbb{C}$-representations of degree e/c for $\mathbf{G}=\mathbf{G}_{-2}$, where $\mathbf{G}_{-2}$ is not isomorphic to $\mathbf{G}_{2}$. They are obtained from those given above for $\mathbf{G}_{2}$ as follows: Let $\eta$ be a primitive 2 nth root of 1 such that $\eta^{2}=\zeta$. Multiply $A$ and $B$ by $\eta^{n c / e}$, then proceed as in the theorem above.

Proof. All that requires checking is that

$$
\mathbf{T}\left(a^{n}\right)=\mathbf{T}\left(b^{n}\right)=\mathbf{T}\left(\omega^{n(n-1) / 2}\right) .
$$

Clearly $\mathbf{T}\left(a^{n}\right)=\mathbf{T}\left(b^{n}\right)=\eta^{n^{2} c / e} \mathbf{I}_{e / c}$. Now $\eta^{n^{2} c / e}=-1$ precisely when $n c / e \equiv 1(\bmod 2)$; otherwise $\eta^{n^{2} c / e}=1$. But $\mathbf{T}\left(\omega^{(n(n-1) / 2}\right)=$ $-\mathbf{I}_{e / c}$ precisely when $e / c$ does not divide $n / 2$, in other words (since $e / c$ does divide $n)$, when $n c / e \equiv 1(\bmod 2)$. Otherwise $\mathbf{T}\left(\omega^{n(n-1) / 2}\right)=\mathbf{I}_{e / c}$. This completes the proof.

We have thus exhibited all irreducible complex representations for these alternative generalizations of the CLE-groups when $r=2$. As we have observed, the groups are abelian when $r=1$. For $r>2$, the groups are central products of smaller groups, and the representations are built up as tensor products of the representations for these smaller groups, just as they were in the previous case. These representations are closely related to projective representations for $(\mathbb{Z} / n \mathbb{Z})^{r}$.

\section{REFERENCES}

[CR] C. W. Curtis and I. Reiner, Representation Theory of Finite Groups and Associative Algebras, Interscience Publishers, J. Wiley and Sons, New York, 1971.

[I] I. M. Isaacs, Character Theory of Finite Groups, Academic Press, Inc., New York, 1976.

[Kw] A. K. Kwasniewski, Clifford- and Grassmann-like algebras-old and new, J.Math. Phys., 26 (1985), 2234-2238.

[LS] T. Y. Lam and T. L. Smith, On the Clifford-Littlewood-Eckmann groups: A new look at periodicity mod 8, Rocky Mountain J. Math., 19 (1989), 749786.

[Li] D. E. Littlewood, Note on the anticommuting matrices of Eddington, J. London Math. Soc., 9 (1934), 41-50. 
[S-I] M. Saeed-ul-Islam, On the projective representations of finite abelian groups, II, J. Math. Phys., 26 (1985), 3033-3035.

[Sm1] T. L. Smith, Some 2-Groups Arising in Quadratic Form Theory and Their Generalizations, Ph.D. Dissertation, Univ. of Calif., Berkeley (1988).

[Sm2] _ Generalized Clifford-Littlewood-Eckmann groups, Pacific J. Math., 149 (1991), 157-183.

[Sm3] _ Decomposition of generalized Clifford algebras, to appear in Quart. J. Math. Oxford.

Received November 20, 1989. This paper was supported in part by NSF Grant DMS8908258. This paper is based in part on the author's doctoral dissertation (University of California, Berkeley, 1988).

The Ohio State University

Columbus, OH 43210 



\section{PACIFIC JOURNAL OF MATHEMATICS EDITORS}

\author{
V. S. VARADARAJAN \\ (Managing Editor) \\ University of California \\ Los Angeles, CA 90024-1555-05 \\ Herbert Clemens \\ University of Utah \\ Salt Lake City, UT 84112 \\ THOMAS ENRIGHT \\ University of California, San Diego \\ La Jolla, CA 92093
}

R. FINN

Stanford University

Stanford, CA 94305

HeRmann FlaschKa

University of Arizona

Tucson, AZ 85721

VAUGHAN F. R. JoNES

University of California

Berkeley, CA 94720

STEVEN KERCKHOFF

Stanford University

Stanford, CA 94305
C. C. MOORE

University of California

Berkeley, CA 94720

MaRTIN SCHARLEMANN

University of California

Santa Barbara, CA 93106

HAROLD STARK

University of California, San Diego

La Jolla, CA 92093

\section{ASSOCIATE EDITORS}
R. ARENS
E. F. BECKENBACH
B. H. NeumanN
F. WolF
K. YosHIDA

\section{SUPPORTING INSTITUTIONS}

UNIVERSITY OF ARIZONA

UNIVERSITY OF BRITISH COLUMBIA

CALIFORNIA INSTITUTE OF TECHNOLOGY

UNIVERSITY OF CALIFORNIA

MONTANA STATE UNIVERSITY

UNIVERSITY OF NEVADA, RENO

NEW MEXICO STATE UNIVERSITY

OREGON STATE UNIVERSITY
UNIVERSITY OF OREGON

UNIVERSITY OF SOUTHERN CALIFORNIA

STANFORD UNIVERSITY

UNIVERSITY OF HAWAII

UNIVERSITY OF TOKYO

UNIVERSITY OF UTAH

WASHINGTON STATE UNIVERSITY

UNIVERSITY OF WASHINGTON 


\section{Pacific Journal of Mathematics}

Vol. 149, No. $1 \quad$ May, 1991

Takao Akahori and Harunori Ameku, On the Romanov kernel and Kuranishi's $L^{2}$-estimate for $\bar{\partial}_{\mathrm{b}}$ over a ball in the strongly pseudo convex

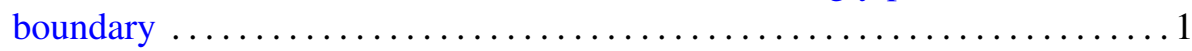

Robert Emile Beaudoin, The proper forcing axiom and stationary set

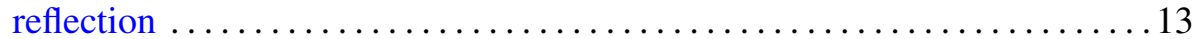

Hans Engler, A matrix Volterra integrodifferential equation occurring in

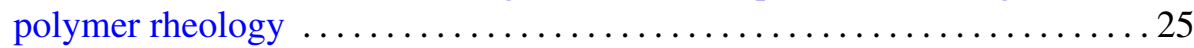

Zhong Ge, On a constrained variational problem and the spaces of

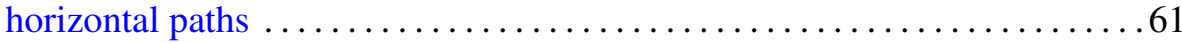

Yutaka Hemmi, Higher homotopy commutativity of $H$-spaces and the

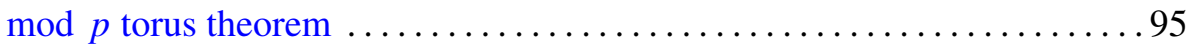

G. D. Johnson, An intrinsic characterization of a class of minimal surfaces

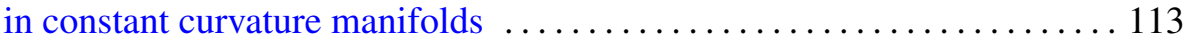

Min Ho Lee, Conjugates of equivariant holomorphic maps of symmetric domains

Jacek Nikiel, H. Murat Tuncali and Edward D. Tymchatyn, On the rim-structure of continuous images of ordered compacta

Tara Lynn Smith, Generalized Clifford-Littlewood-Eckmann groups . . . . . 157

Tara Lynn Smith, Generalized Clifford-Littlewood-Eckmann groups II:

Linear representations and applications 185 